\title{
Multifaceted Approach to Analyzing the Pathogenesis of Cardiovascular Disease
}

\author{
Motoaki Sano, MD, PhD
}

\begin{abstract}
Heart failure is a common clinical feature of advanced cardiovascular disease that carries a high mortality risk. This review focuses on my journey seeking to understand the pathogenesis of heart failure and on how to translate experimental findings into the clinic. (Circ $J$ 2012; 76: 2521-2529)
\end{abstract}

Key Words: Cardiac hypertrophy; Cardiac metabolism; Cardiac remodeling; Myocardial infarction; Oxidative stress

$\mathbf{H}$ eart failure (HF) affects 6 million patients in the United States, with more than 600,000 people newly diagnosed each year. Although there are no definitive data yet available on the prevalence of HF in Japan, the incidence of cardiovascular disease (CVD) in Japan remains lower than in Europe and America. However, the increasingly westernized diet and advancing age of the Japanese population might see the incidence of HF approaching that reported in the United States.

Cardiac hypertrophy is a common feature of the failing myocardium in patients with hypertension, myocardial infarction (MI), and valvular heart diseases, and increased ventricular mass is an independent risk factor for cardiovascular mortality in humans. Therefore, blunting hypertrophic growth might be beneficial to function or prognosis in these patients. Blocking angiotensin II (AngII) could be especially effective in reversing left ventricular hypertrophy $(\mathrm{LVH})$. Herein I review the mechanism of AngII-induced cardiomyocyte hypertrophy.

Hypertrophic signaling cascades resemble a complex web, affecting initiation of transcription, mRNA stability, or translation, and leading to fetal gene activation, myocyte enlargement, or both. I also review our discovery of a novel hypertrophic signaling cascade that enhances transcriptional elongation.

Oxidative stress is involved in the pathogenesis of $\mathrm{HF}$ and aging of the heart, thus the risk of developing HF dramatically increases with age. It is therefore crucial to understand how the heart copes with oxidative stress. Because cardiomyocytes rely primarily on the energy produced by mitochondrial oxidative phosphorylation and mitochondria produce abundant reactive oxygen species (ROS) as a byproduct of oxidative phosphorylation, the heart is especially prone to oxidative damage. Therefore, heart is equipped with multiple layers of antioxidant defense mechanisms. ROS attack polyunsaturated fatty acids of the cell membrane and trigger lipid peroxidation, which results in the generation of aldehydes. Aldehydes in turn upregulate the expression levels of antioxidant enzymes via a nuclear factor erythroid 2-related factor 2 (Nrf-2)-dependent pathway. If aldehyde accumulation chronically exceeds their detoxification, the heart alters its metabolic pathway in order to produce higher levels of intracellular reductants, such as reduced glutathione via the eukaryotic initiation factor $2 \alpha$ subunit (eiF2 $\alpha$ )-activating transcription factor-4 (ATF4) pathway, and reestablish homeostasis.

Despite an impressive decline in deaths attributable to acute MI, the incidence of HF has paradoxically increased. The combination of reduced acute infarct mortality because of efficient acute care and insufficient options to treat infarct survivors contribute to the increased HF prevalence. Left ventricular (LV) systolic dysfunction develops during hospitalization or within 30 days of acute MI. LV remodeling leads to chronic HF and is the main determinant of morbidity and mortality after MI. At the present time, therapeutic options to prevent $L V$ remodeling are limited. The unmet medical needs in the present era of primary percutaneous coronary intervention (PCI) after MI are the prevention of ischemia-reperfusion (IR) injury and subsequent targeted intervention against the MI-induced inflammatory response. I review how to reduce infarct volume and mitigate post-MI cardiac remodeling.

\section{Cross-Talk Between Fibroblasts and Myocytes in Angll-Induced Cardiac Hypertrophy}

In 1987, large-scale double-blind randomized trials demonstrated the survival benefits of using angiotensin-converting enzyme (ACE) inhibitors in patients with LV systolic dysfunction. ${ }^{1}$ Consequently, blocking AngII activity became the gold standard for treating systolic HF. Ventricular hypertrophy develops in response to numerous forms of cardiac stress and often leads to HF. LVH is a fundamental manifestation of preclinical CVD and an independent risk factor for cardiovascular mortality, ${ }^{2}$ and reversal of LVH has possible prognostic benefits that are independent of blood pressure. Both clinical and experimental evidence have demonstrated that AngII is associated with the development of LVH. However, the question remains as to the mechanism of AngII-induced cardiac hypertrophy.

Received August 21, 2012; accepted September 12, 2012; released online September 29, 2012

Department of Cardiology, Keio University School of Medicine, Tokyo, Japan

Mailing address: Motoaki Sano, MD, PhD, Department of Cardiology, Keio University School of Medicine, 35 Shinanomachi, Shinjuku-ku,

Tokyo 160-8582, Japan. E-mail: msano@a8.keio.jp

ISSN-1346-9843 doi:10.1253/circj.CJ-12-1063

All rights are reserved to the Japanese Circulation Society. For permissions, please e-mail: cj@j-circ.or.jp 


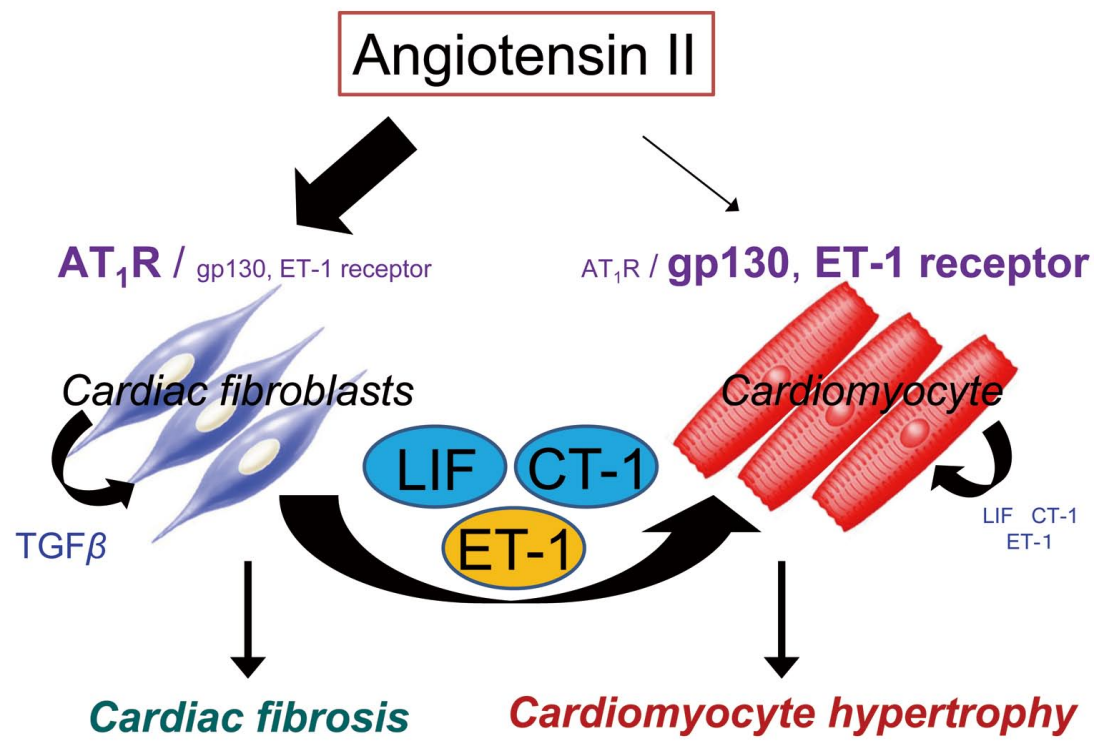

Figure 1. Mechanism of angiotensin II (Angll)-induced cardiac hypertrophy. The cross-talk between fibroblasts and cardiomyocytes via Leukemia inhibitor factor (LIF), cardiotrophin-1 (CT-1), and endothelin1 (ET-1) plays an important role in Angll-induced cardiomyocyte hypertrophy. AT1R, type 1 Angll receptor.

Although cardiomyocytes are the predominant cellular constituent of the heart mass, cardiac fibroblasts outnumber them 2 -fold. Because cardiomyocytes express less than $10 \%$ of the number of type 1 AngII receptors (AT1R) expressed in cardiac fibroblasts, it is difficult to explain why cardiomyocytes respond effectively to ACE inhibitors and AT1R blockers (ARBs). We knew, empirically, that AngII-induced cardiomyocyte hypertrophy is stimulated in culture by increasing the number of cardiac fibroblasts. Accordingly, the discrepancy between the low levels of AT1R in cardiomyocytes and the high effectiveness of ACE inhibitors and ARBs on cardiomyocyte hypertrophy could be explained by the cross-talk of paracrine factors between cardiac fibroblasts and cardiomyocytes.

Leukemia inhibitor factor (LIF), which is also known as a cholinergic neuronal differentiation factor, belongs to the interleukin (IL)-6 family of cytokines. LIF rapidly activates the JAK/STAT pathway via the gp130 receptor, which is abundantly expressed in cardiomyocytes, to induce cardiomyocyte hypertrophy. ${ }^{3-5}$ Interestingly, AngII, which transduces signals via the G-protein-coupled AT1R, biphasically activates the JAK/STAT pathway and induces delayed phosphorylation of STAT1/STAT3 in cardiomyocytes. This delayed phosphorylation of STATs was also stimulated in culture by increasing the number of cardiac fibroblasts. Thus, we hypothesized that AngII induced LIF in cardiac fibroblasts and that LIF-activated gp130-linked signaling contributed to AngII-induced cardiomyocyte hypertrophy. In cultured cardiac fibroblasts, AngII induced IL-6, LIF, and cardiotrophin-1 (CT-1). Conditioned medium prepared from Ang I-stimulated cardiac fibroblasts could phosphorylate gp130 and STAT3 in cardiomyocytes, and induce cardiomyocyte hypertrophy. These effects are partially blocked by anti-gp130 blocking antibody or antisense oligonucleotides against LIF or CT-1. Collectively, evidence from mine ${ }^{6,7}$ and other groups in $\mathrm{Japan}^{8,9}$ demonstrates that the cross-talk between cardiac fibroblasts and cardiomyocytes via paracrine factors is important for AngII-induced cardiomyocyte hypertrophy (Figure 1).

Consistent with this result, LIF and CT-1 are strongly upregulated in the hypertrophied ventricles of Dahl salt-sensitive rats. Interestingly, these same factors secreted from the hypertrophied myocardium induce cholinergic differentiation of the cardiac sympathetic nerves via a gp130-linked signaling. ${ }^{10,11}$ This could explain the discrepancy between the excess sympathetic nerve activation and the paradoxical reduction in norepinephrine uptake in the cardiac sympathetic nerves of HF patients.

\section{RNA Polymerase II Kinase: Potential Target for Blunting Hypertrophic Growth}

Clinical studies have demonstrated that increased ventricular mass is an independent risk factor for cardiovascular mortality in humans. Therefore, blunting hypertrophic growth might be beneficial to function or prognosis in CVD. What remains unproven is which pathways or signals hold the greatest potential for therapeutic benefit.

The hypertrophic phenotype is characterized by increased myocyte size via a global increase in the RNA and protein content per cell, as well as transcriptional reprogramming that can resemble a fetal gene program. Signaling pathways that activate this hypertrophy-associated fetal gene program have been vigorously studied; ${ }^{12}$ however, the mechanisms that govern the increased myocyte size of hypertrophic growth remain unknown.

In eukaryotes, transcription of protein-coding genes is catalyzed by RNA polymerase II (pol II), and phosphorylation of pol II in its carboxy-terminal domain (CTD) is an essential mediator of mRNA production. In mammals, the CTD comprises 52 repeats of an evolutionally conserved serine-rich heptapeptide, Tyr-Ser-Pro-Thr-Ser-Pro-Ser. Hypophosphorylated pol II is the form recruited to promoters for transcript initiation; the CTD becomes extensively phosphorylated, primarily at Ser2 and Ser5 of the heptapeptide repeat, to overcome proximal promoter pausing and confer productive transcript elongation. In this way, CTD phosphorylation is required for productive transcription elongation and pre-mRNA processing, and thus is a hallmark of the actively transcribed pol II. Two cyclin-dependent kinases (CDKs), CDK7 and CDK9, regulate transcription by phosphorylating the CTD. CDK9 
forms positive transcription elongation factor-b with its partner cyclin T, while the CDK7/cyclin H/MAT-1 complex is a kinase subunit of TFIIF.

At first, my group monitored pol II phosphorylation and the CTD kinases in mouse models of cardiac hypertrophy. ${ }^{13}$ In calcineurin cardiac overexpression models, pol II was 2-fold more phosphorylated than wild-type (WT) non-transgenic (TG) littermates. In accordance with the enhanced phosphorylation of pol II, both CDK7 and CDK9 activities increased in the TG myocardium. In contrast to the activation of both CDK7 and CDK9 in chronic cardiac hypertrophy models, an acute mechanical load provoked only the activation of Cdk9. To examine the CTD kinase activation under more defined conditions, we then used primary cultured cardiomyocytes. Endothelin-1 (ET-1)-induced pol II phosphorylation was detected at $5 \mathrm{~min}$, and peaked at $15 \mathrm{~min}$. As observed with acute pressure overload in vivo, only the CDK9-associated CTD kinase activity increased, without a parallel change in CDK7 activity. No increase occurred in the levels of Cdk9 or cyclin T. To establish more directly which CTD kinases mediate ET-1-induced phosphorylation of pol II, we transfected catalytically inactive, dominant-negative CDK9 and CDK7 by viral gene transfer. Dominant-negative CDK9 specifically inhibited ET-1-induced pol II phosphorylation, with no measurable effect on Cdk7 kinase activity. Conversely, dominant-negative CDK7 suppressed CDK7 activity, but had no effect on ET-1-induced pol II phosphorylation. These results indicated that CDK9 is the essential CTD kinase for ET-1-induced pol II phosphorylation. They also showed that CDK9 activity mediates cardiac growth, because the dominant-negative CDK9 markedly inhibited ET1-induced cardiac myocyte enlargement, whereas dominantnegative $\mathrm{Cdk} 7$ did not. To investigate whether activation of CDK9 promotes growth in intact hearts, we made cyclin T1 TG mice, resulting in 2 lines with cyclin T1 levels and CDK9 kinase activity increased 2- and 6-fold, respectively. In proportion to the CDK9 kinase activity, heart/body weight ratio increased $21 \%$ and $42 \%$, respectively.

Next, we asked how do the hypertrophic signals activate CDK9? Paradoxically, while both CDK9 activity and CDK9dependent phosphorylation of pol II increase, neither Cdk9 nor cyclinT expressions increased, in vitro or in vivo. Furthermore, no increase occurred in the assembly of cyclinT/CDK9. At that time, 2 independent groups identified 7SK, a small nuclear RNA, as a component of the Cdk9/cyclinT complex. 7SK suppresses Cdk9 kinase activity and inhibits productive transcription elongation. ${ }^{14,15}$ As a first step to test for this inhibitory RNA in endogenous cardiac cyclinT/CDK9 complexes, we treated Cdk9 immune complexes with RNaseA, then assayed for activity towards CTD. RNaseA increased CDK9 kinase activity in control cells to levels even higher than ET-1. Next, we proved the association of 7SK with cardiac cyclin T/CDK9 complexes by reverse transcription polymerase chain reaction (RT-PCR) and verified its identity by cDNA sequencing. No 7SK RNA was associated with CDK7, and no PCR product was formed in the absence of reverse transcriptase. To determine whether hypertrophic stimuli resulted in the release of 7SK from cardiac CDK9/cyclinT complexes, we tested for 7SK physically associated with CDK9/ cyclinT with and without prior ET-1 treatment. ET-1 caused the rapid loss of CDK9-associated 7SK within $15 \mathrm{~min}$. We proved these findings using 3 antibodies against the CDK9/cyclinT complex. To test if interference with 7SK snRNA would suffice to induce cardiac CDK9 activity and RNA synthesis, we used antisense oligonucleotides. Loss of 7SK snRNA markedly increased endogenous CDK9 activity, and induced an increase in $\left[{ }^{3} \mathrm{H}\right]$-uridine incorporation.

In conclusion, Cdk9 kinase activity is increased in divergent models of cardiac hypertrophy. Hypertrophic triggers activate CDK9 through dissociation of the 7SK small nuclear RNA complex, and then stimulate pol II activities through the phosphorylation of pol II. Activation of CDK9 stimulates productive mRNA synthesis and suffices to induce cardiac hypertrophy (Figure 2A). Therefore, CDK9 inhibition could be a novel target in the management of deleterious cardiac hypertrophy. ${ }^{13,16,17}$

Hypertrophy initially allows the heart to adapt to workload, but prolonged hypertrophy eventually causes HF. CDK9 activity is increased in response to hypertrophic stimuli and is continuously elevated in failing myocardium. My group confirmed the significant increase of CDK9 activity in human heart samples from patients suffering from dilated cardiomyopathy.$^{18}$ However, whether this prolonged activation of CDK9 is adaptive or adverse remained unanswered. As already noted, cardiac-specific overexpression of cyclin T1 causes cardiac hypertrophy corresponding to increased CDK9 activity. Because cyclin T1 TG hearts seemed benign under basal conditions, we stressed them by subjecting them to pressure overload. Aortic-banded cyclin T1 mice show increased concentric hypertrophy, systolic dysfunction, and high postoperative lethality, but most importantly, increased apoptosis shown by cleavage of caspase- 3 and an increased prevalence of TUNELpositive cardiomyocytes.

To seek a molecular signature for the susceptibility to apoptotic cardiomyopathy conferred by CDK9 activation, we compared microarray expression profiles of ventricular myocardium from cyclin T1 TG, Gq TG, and non-TG mice. Because CDK9 activity promotes transcription elongation, persistent Cdk9 activation might be expected to cause a global increase in cardiac mRNA synthesis with no difference in the mRNA expression profile. Instead, a more nuanced profile was seen. Despite the fact that cyclin T1 mice had an increase in cardiac mass to the same extent as in $\mathrm{Gq}$ mice, little or no induction occurs for common hypertrophic markers, such as atrial and B-type natriuretic peptides, $\alpha$-skeletal action, and $\beta$-MHC. Surprisingly, many genes were suppressed by CDK9 activation. The largest cluster of these genes identified by the microarray survey comprised those for mitochondrial function including enzymes for respiratory chain complexes, the tricarboxylic acid cycle, $\beta$-oxidation of fatty acids, antioxidant enzymes, mitochondrial ribosomal proteins, and mitochondrial transcription factors. Impaired mitochondrial function would conceivably explain why cyclin $\mathrm{T} 1$ mice decompensate dramatically when challenged with cardiac stress. We speculated that the coordinated downregulation of these genes might result from impaired function or expression of a limiting transcriptional activator. An especially apt candidate was peroxisome proliferator-activated (PPAR)- $\gamma$ coactivator-1 (PGC-1), a master regulator of mitochondrial function and biogenesis.

The expression of mitochondrial proteins encoded by nuclear and mitochondrial genomes is coordinately regulated by PGC-1, which orchestrates a transcriptional network through binding nuclear hormone receptors and other transcription factors. PGC-1 induces mitochondrial transcription factor A via coupling with nuclear respiratory factor (NRF)-1, which in turn is essential for mitochondrial DNA replication and transcription. PGC-1 is highly expressed in mitochondria-rich tissues that have high energy demands, such as the heart. Notably, PGC-1 expression was downregulated in response to pressure overload. The downregulation of PGC-1 correlated well with the decreased expression in nuclear- and mitochon- 


\section{A}
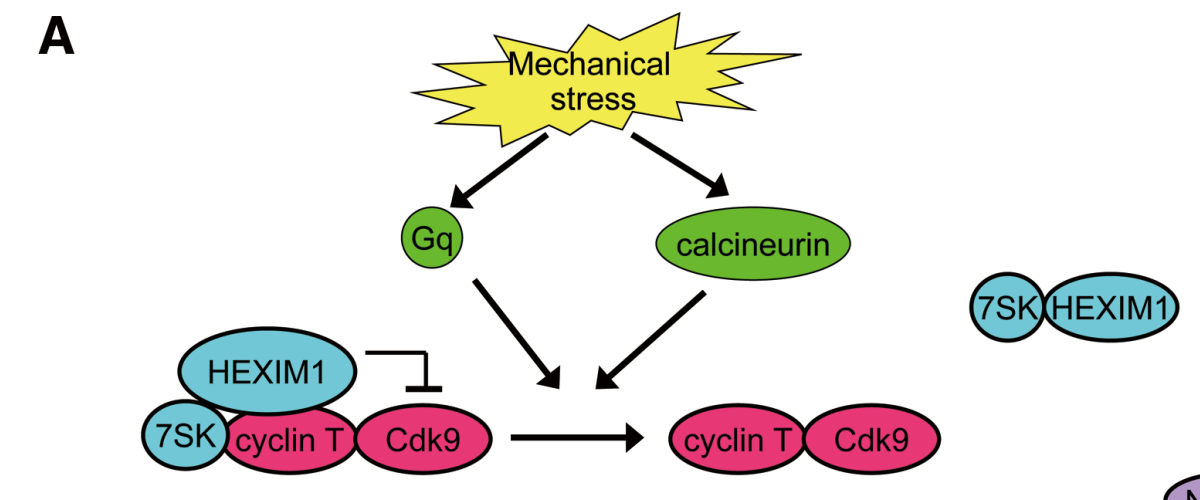

5
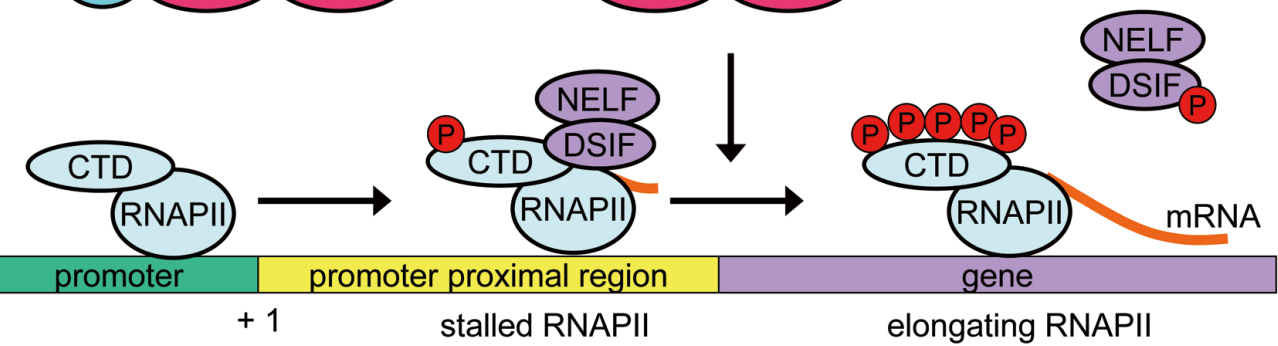

B

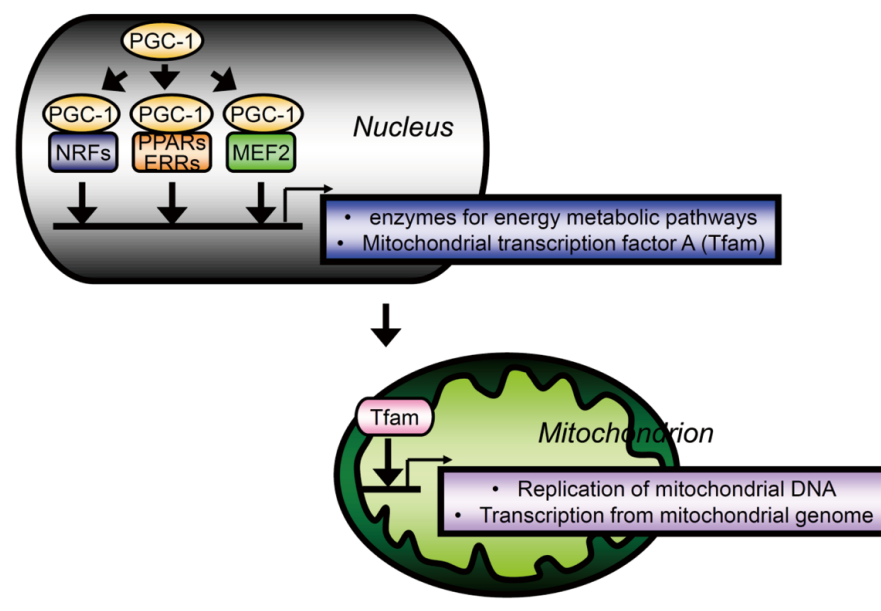

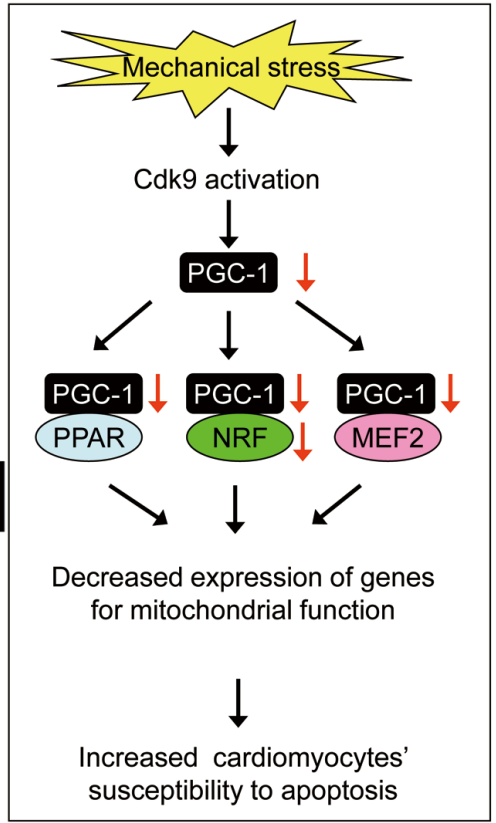

Figure 2. RNA polymerase II kinase CDK9 is a potential target for blunting hypertrophic growth. (A) Hypertrophic triggers activate CDK9 through dissociation of its endogenous inhibitor, the 7SK small nuclear RNA complex, and then stimulate RNA polymerase II activities through the phosphorylation of RNA polymerase II. (B) Chronic activation of CDK9 causes defective mitochondrial function via diminished PGC-1 transcription, and resulting susceptibility to apoptotic cardiomyopathy. CDK, cyclin-dependent kinase; CTD, carboxy-terminal domain; PGC, peroxisome proliferator-activated (PPAR)- $\gamma$ coactivator-1; NRF, nuclear factor erythroid 2-related factor. 
dria-encoded genes for oxidative energy metabolism and ROSdetoxifying enzymes. ${ }^{19-21}$

The molecular mechanisms underlying the loss of PGC-1 expression in cardiac hypertrophy are poorly understood. Chronic activation of CDK9 causes defective mitochondrial function via diminished PGC-1 transcription, resulting in susceptibility to apoptotic cardiomyopathy (Figure 2B). CDK9 inhibitors could therefore be used in the management of patients with $\mathrm{HF}$ in stages B through D.

\section{How to Deal With Oxidative Stress?}

It is well accepted that the highest possible levels of perceived stress correlate with the early onset of CVD. For example, chronic stress facilitates plaque formation in the coronary arteries, and superimposed acute stress often triggers plaque rapture and thrombosis, leading to MI, a major cause of sudden death. Conversely, appropriate (mild to moderate) stressors have been shown to promote health. For example, physical exercise is known to decrease the risk of premature mortality in general and the risk of coronary artery disease, hypertension, cancer, and diabetes mellitus in particular. Intense muscular contractile activity through exercise results in "oxidative stress" and excess vitamin C supplements decrease training efficacy. However, despite oxidative stress being associated with CVD, the antioxidant supplement failed to prevent the incidence of CVD and all causes of mortality, indicating that oxidative stress may not be completely harmful. Therefore, it is very important to understand how biological systems respond to low levels of oxidative stress and the implications of such findings for disease pathogenesis and control.

ROS attack polyunsaturated fatty acids of the cell membrane, triggering lipid peroxidation and leading to the generation of $\alpha, \beta$-unsaturated aldehydes, such as 4-hydroxy-2-nonenal (4-HNE). There is compelling evidence that high-dose aldehydes are responsible for much of the damage elicited by ROS, whereas low doses of aldehydes stimulate stress resistance pathways to achieve cardioprotection. My group ${ }^{22}$ found that in cultured cardiomyocytes, 4-HNE is cytotoxic at higher concentrations, but has no appreciable adverse effect at lower concentrations. By contrast, pretreatment with a low dose of 4-HNE primed cardiomyocytes in culture to become resistant to cytotoxic, high-dose concentrations of 4-HNE. Next, we investigated whether systemic administration of 4-HNE primes the heart to become resistant to oxidative injury. 4-HNE was injected via the retro-orbital vein and we first examined whether sufficient reactive 4-HNE reaches the heart after systemic administration. Using antibodies specific for 4-HNE adduct proteins, we found that the level of 4-HNE in the heart was increased at $60 \mathrm{~min}$ after administration. We also demonstrated that cardiac Nrf2 is activated at 60 min after systemic administration of 4-HNE. Nrf2 has been implicated as a key transcription factor that induces the expression of antioxidant genes. Thus, the systemic administration of 4-HNE significantly upregulated the levels of mRNA for antioxidant enzymes. 4HNE pretreatment significantly improved functional recovery after IR in Langendorff-perfused mouse hearts. As we expected, no cardioprotective effect of systemic administration of 4-HNE was observed in the Nrf-2 knockout mice. These results indicated that low-dose aldehyde confers rapid cardioprotection against subsequent lethal oxidative injury via Nrf2dependent antioxidant gene expression.

Aldehydes accumulate with aging. Therefore, we next asked whether cardioprotection could be achieved within the setting of chronic exposure to aldehydes throughout life? To test this, we paid attention to 1 molecule, ALDH2, which is a major aldehyde detoxifying enzyme in the mitochondria. There is a polymorphism specific to North-East Asians in the ALDH2 gene. ${ }^{23} \mathrm{~A}$ mutant allele, ALDH $2 * 2$, has a single point mutation of the active ALDH $2 * 1$ gene, acting as a dominant-negative gene. ALDH2 acts as homo or hetero-tetramer and all tetramers containing at least $1 \mathrm{ALDH} 2 * 2$ subunit are inactive.

We created a loss-of-function model of Aldh 2 by overexpressing Aldh $2 * 2$ under the control of the CAG (cytomegalovirus enhancer, chicken $\beta$-actin promoter, rabbit $\beta$-globin polyA signal) promoter. A cell fractionation study localized the Aldh $2 * 2$ protein to the mitochondria. The forced expression of Aldh2*2 impaired Aldh activity against aliphatic aldehydes, including 4-HNE (Figure 3A). We suspect that Aldh2*2 inactivates not only Aldh2, but also other Aldh subfamilies, presumably by forming heterotetramers. Consistent with this, we found that levels of 4-HNE adduct proteins were increased in the mitochondrial fraction, but not in the cytosolic fraction of the TG hearts. These findings indicated that Aldh2*2-TG hearts were exposed to higher levels of mitochondrial aldehydes than the WT hearts. ${ }^{24}$ Ultrastructural analysis by transmission electron microscopy revealed electron-dense deposits on an impaired membrane structure in the mitochondria. Interestingly, echocardiographic examination revealed that LV function in the loss-of-function mice was equivalent to that in WT littermates until at least 2 years of age. These results indicated that the TG heart adapts to persistent mitochondrial oxidative stress at least under unstressed conditions. Notably, Aldh $2 * 2$ TG hearts are resistant to exogenous oxidative stress elicited by IR injury. These findings indicate that cardioprotection can be achieved within the setting of chronic exposure to aldehydes.

We then investigated the possible mechanism of cardioprotection. Surprisingly, expressions of most of the major antioxidant enzymes, such as catalase, superoxide dismutase, and GPX, were unchanged in the Aldh2*2 TG hearts. Instead, there was an upregulation of genes encoding enzymes involved in amino acid biosynthesis and transport in the TG hearts. We therefore hypothesized that optimization of cardiac metabolic pathways is a key mechanism of cardioprotection under lifelong mitochondrial oxidative stress. To verify the changes in glucose metabolism, we performed in vivo pulse-chase analysis of ${ }^{13} \mathrm{C}$-labeled glucose in Langendorff-perfused hearts. ${ }^{13} \mathrm{C}$-Labeled metabolites were quantified by CE-MS/MS $5 \mathrm{~min}$ after administration of ${ }^{13} \mathrm{C}$-labeled glucose. ${ }^{13} \mathrm{C}$-labeled intermediate metabolites of the glycolysis were higher in TG hearts than in Wt hearts, indicating an enhanced glycolytic pathway in the Tg hearts. From this in vivo pulse-chase analysis of ${ }^{13} \mathrm{C}$ labeled glucose, we also found that glucose biotransformation was shifted from glycolysis towards the pentose phosphate pathway to generate NADPH and amino acid serine biosynthesis. Genes upregulated in the TG hearts were 3-phosphoglycerate dehydrogenase (Phgdh), phosphoserine aminotransferase (Psat1), and phosphoserine phosphatase (Psph), all of which are involved in the 3-step conversion of 3-phosphoglycerate (a glycolytic intermetabolite) to serine. Serine hydroxymethyltransferase (Shmt) 1/2 catalyzes the conversion of serine to glycine, and serine is also used for cysteine synthesis in the trans-sulfuration pathway. Glutathione (GSH) is synthesized from 3 amino acids, cysteine, glycine, and glutamate. We speculate that activation of serine biosynthesis plays a key role in the maintenance of higher levels of GSH biosynthesis for cardioprotection (Figure 3B). In fact, the GSH levels were increased in Aldh2*2 TG hearts compared with WT controls. We identified that the eIF2 $\alpha$-ATF4 pathway is a key mito- 

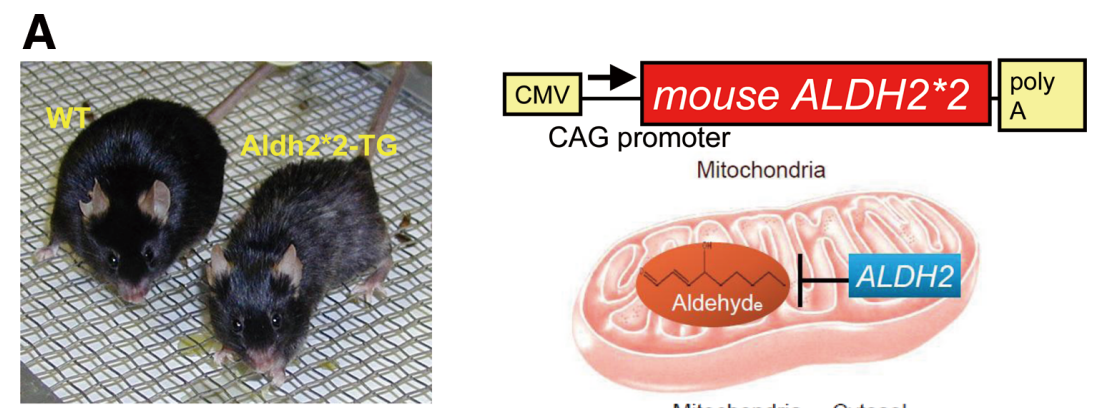

CAG promoter

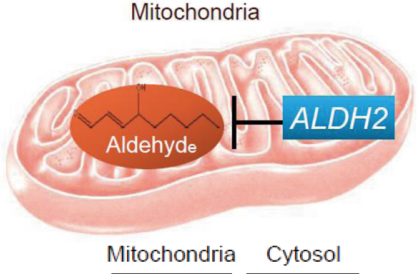

\begin{tabular}{|c|c|c|}
\hline & \multicolumn{2}{|c|}{ ALDH activity } \\
\hline substrate & Wt & $\mathrm{Tg}$ \\
\hline Acetaldehyde & 7.31 & 5.35 \\
\hline Butyraldehyde & 7.75 & 5.59 \\
\hline Hexanal & 9.40 & 5.78 \\
\hline 4-HNE & 5.38 & 3.52 \\
\hline Trans-2-Nonenal & 6.37 & 4.44 \\
\hline propionaldehyde & 8.87 & 6.13 \\
\hline
\end{tabular}
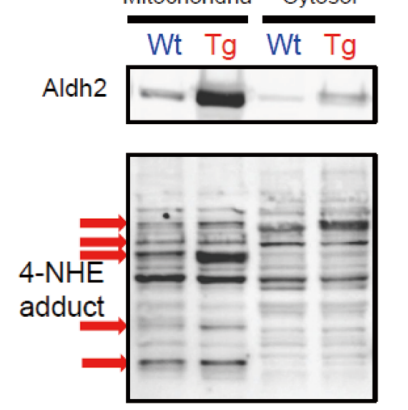

B

\section{Glycolytic pathway}

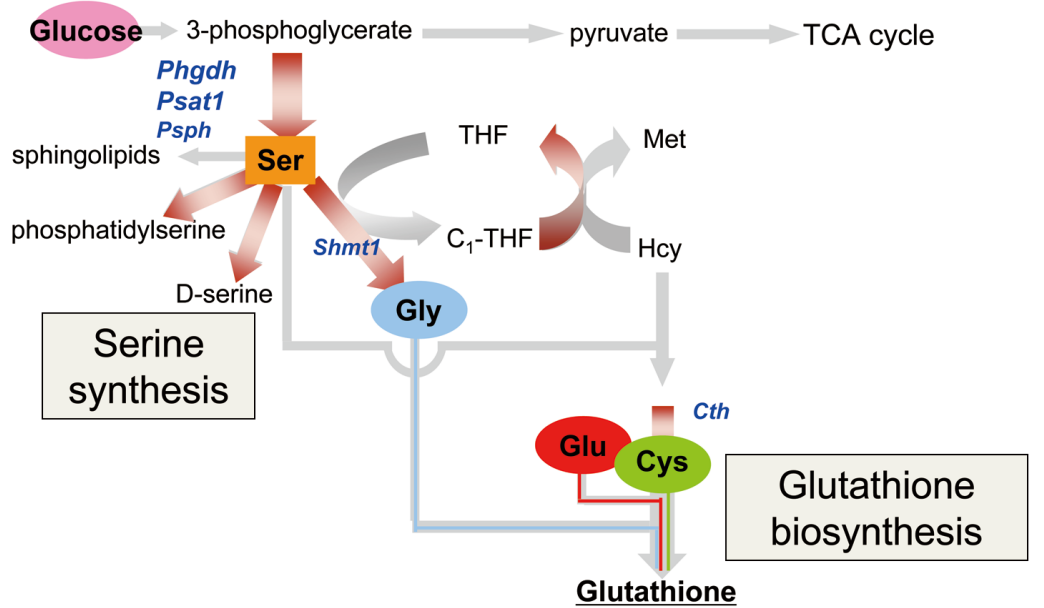

\section{C}

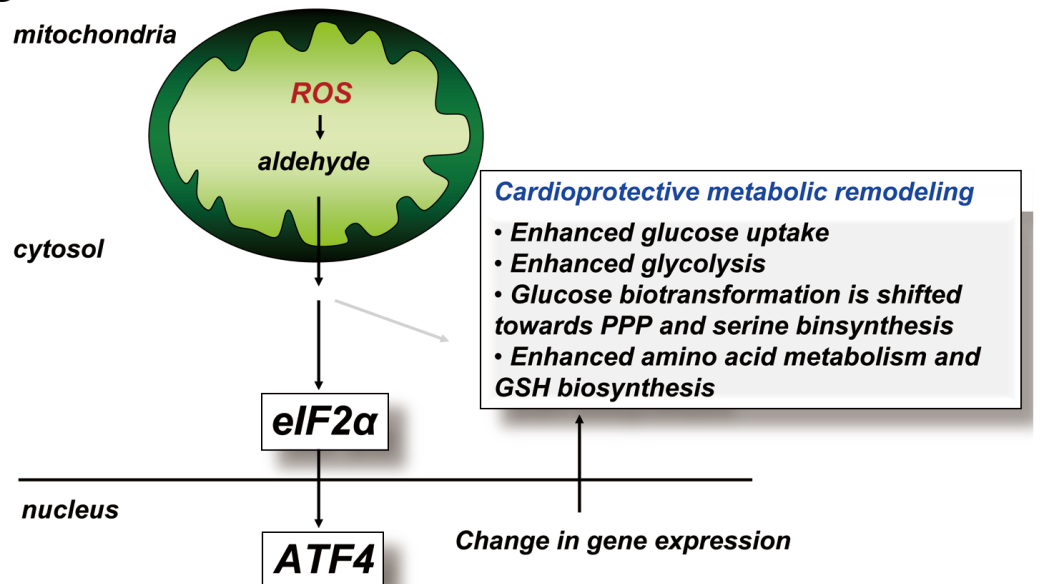

Figure 3. Intrinsic capacity of the heart to deal with chronic mitochondrial oxidative stress. (A) Aldh2*2 transgenic mice represent a loss-of-function model of aldehyde (Aldh) activity. (B) Metabolic map depicting that activation of serine (Ser) biosynthesis plays a key role in the maintenance of higher levels of glutathione biosynthesis for cardioprotection. (C) The elF2a-ATF4 pathway is a key mitochondrial retrograde signal in response to chronic mitochondrial oxidative stress. 4-HNE, 4hydroxy-2-nonenal; ATF4, activating transcription factor-4; CAG, cytomegalovirus enhancer, chicken $\beta$-actin promoter, rabbit $\beta$-globin polyA signal; eiF2a, eukaryotic initiation factor 2 a subunit; GSH, glutathione; Gly, glycine; Phgdh, 3-phosphoglycerate dehydrogenase; PPP, Psat1, phosphoserine aminotransferase; Psph, phosphoserine phosphatase; ROS, reactive oxygen species; TCA, tricarboxylic acid; TG, transgenic; WT, wild-type. 


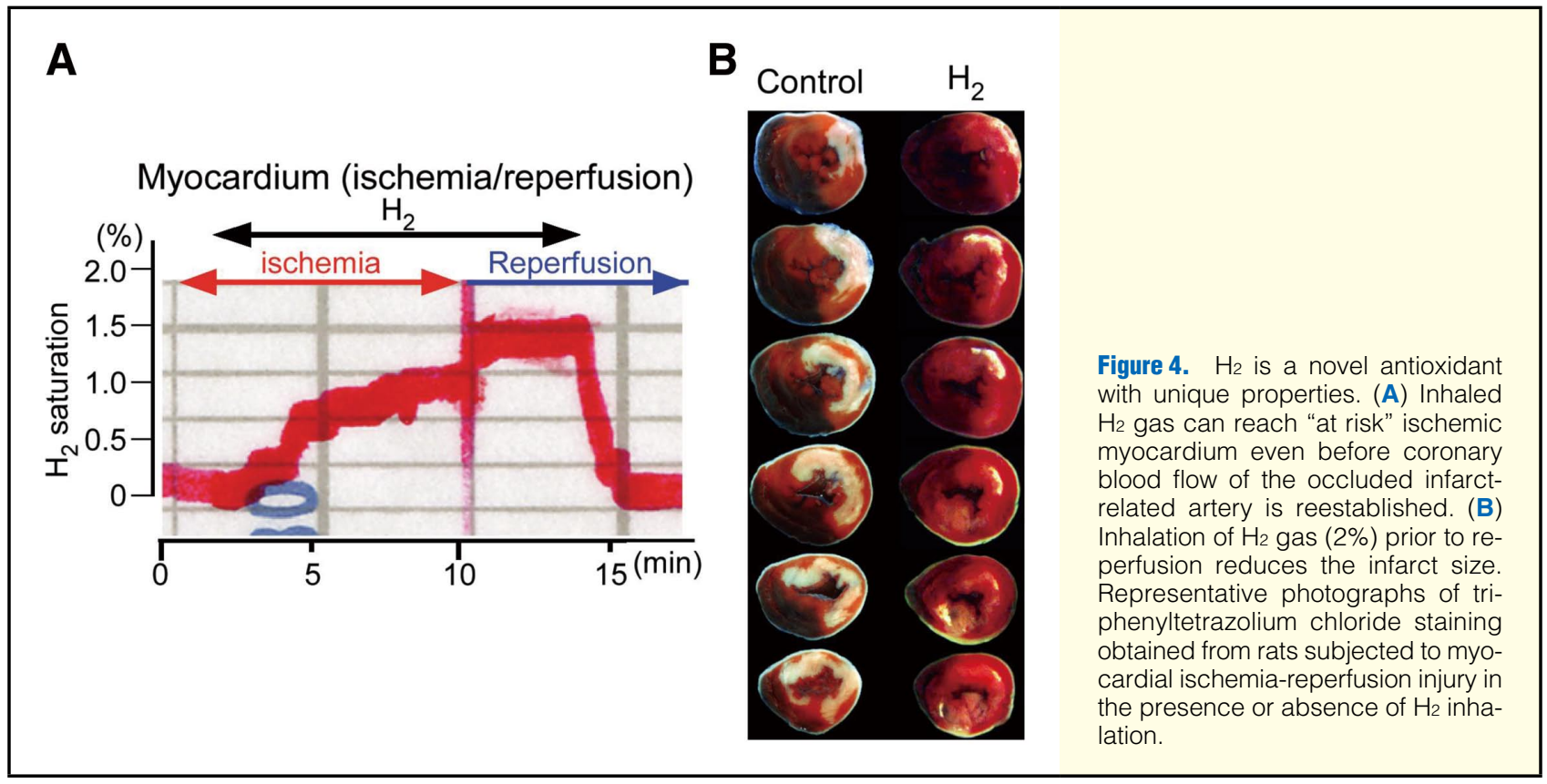

chondrial retrograde signaling response to chronic mitochondrial oxidative stress (Figure 3C). How this retrograde response to mitochondrial oxidative stress is affected by disease and aging is an important area for future research.

Low-dose oxidative stress is beneficial in both the acute and chronic situations. Aldehyde-mediated cardioprotection is accomplished by the activation of 2 transcription factors, $\mathrm{Ntf} 2$ and ATF4, at different time points. ${ }^{25,26}$ Therefore, activators of Nrf2 and ATF4 are potentially useful therapeutic tools with which to fight cardiac oxidative stress.

\section{Promising Strategies to Further Decrease Infarct Size and Prevent Post-MI HF}

$\mathrm{MI}$ is the most frequent cause of $\mathrm{HF}^{27}$ and the MI infarct volume and subsequent adverse LV remodeling (dilation and fibrosis) determine the degree of LV dysfunction. Timely revascularization of ischemic myocardium followed by standard therapy with renin-angiotensin-aldosterone inhibitors and $\beta$ blockers can alleviate post-MI remodeling. However, over the past decade, a paradoxical increase in the incidence of $\mathrm{HF}$ within the 30-day post-MI period has been observed. ${ }^{28}$ Therefore, innovative treatment to prevent progressive LV dysfunction during hospitalization following $\mathrm{MI}$ is an important unmet medical need, and we propose 2 strategies for doing this: (1) reduce IR injury; and (2) immunomodulatory therapy to alleviate adverse $\mathrm{LV}$ remodeling.

Studies in animal models of acute MI show that reperfusion injury accounts for up to $50 \%$ of the final size of a myocardial infarct. Therefore, intervention to alleviate reperfusion injury at the time of coronary recanalization has been considered a promising strategy to further decrease infarct size and improve the prognosis after MI. ${ }^{29}$ The accelerated generation of ROS by reperfusion of the ischemic myocardium is a potential mediator of reperfusion injury, and to this end, many attempts have been made to inhibit ROS production to limit the extent of reperfusion injury. However, administering ROS scavengers at the time of reperfusion has produced conflicting results, which can be explained by the "Jekyll and Hyde" characteris- tics of ROS in ischemia-reperfused hearts. The majority of detrimental effects associated with lethal reperfusion injury are attributed to the hydroxy radical $(\cdot \mathrm{OH})$, which has the highest oxidative energy. By comparison, the superoxide anion radical $\left(\mathrm{O}_{2}{ }^{-} \cdot\right)$ and hydrogen peroxide $\left(\mathrm{H}_{2} \mathrm{O}_{2}\right)$ have less oxidative energy and are implicated as crucial signaling components in the establishment of favorable tolerance to oxidative stress upon IR. Consequently, nonselective inhibition of all ROS can be harmful.

Molecular hydrogen $\left(\mathrm{H}_{2}\right)$ is a novel antioxidant with certain unique properties. ${ }^{30}(1) \mathrm{H}_{2}$ is permeable to cell membranes and can target organelles, including mitochondria and nuclei. (2) $\mathrm{H}_{2}$ specifically quenches exclusively detrimental ROS, such as $\cdot \mathrm{OH}$ and peroxynitrite $\left(\mathrm{ONOO}^{-}\right)$, while maintaining the metabolic oxidation-reduction reaction and other less potent ROS, such as $\mathrm{O}_{2}^{-} \cdot \mathrm{H}_{2} \mathrm{O}_{2}$, and nitric oxide (NO-). (3) Inhalation of hydrogen $\left(\mathrm{H}_{2}\right)$ gas limits the infarct volume of brain by reducing IR injury in rodents. When translated into clinical practice, this therapy must be most frequently applied in the treatment of patients with acute MI, because angioplastic recanalization of the infarct-related occluded coronary artery is routinely performed. Therefore, my group investigated whether $\mathrm{H}_{2}$ gas confers cardioprotection against IR injury in rats.

As a first step to confirm the efficacy of $\mathrm{H}_{2}$ gas, we confirmed that inhaled $\mathrm{H}_{2}$ gas is rapidly transported and can reach "at risk" ischemic myocardium even before coronary blood flow of the occluded infarct-related artery is reestablished (Figure 4A) ${ }^{31}$ Inhalation of $\mathrm{H}_{2}$ gas at incombustible levels during IR reduces infarct size without altering hemodynamic parameters, thereby preventing deleterious LV remodeling (Figure 4B). Thus, inhalation of $\mathrm{H}_{2}$ gas is a promising strategy to alleviate IR injury coincident with recanalization of coronary artery. The efficacy of $\mathrm{H}_{2}$ gas was also confirmed in a canine model of IR injury by the Asanuma Group. ${ }^{32}$ We thus decided to perform a first-in-human study to evaluate the safety and efficacy of inhalation of $\mathrm{H}_{2}$ gas during PCI in patients with acute MI (UMIN000006825). This study has just started at Keio University School of Medicine.

Adverse LV remodeling after MI leads to chronic $\mathrm{HF}$ and is 

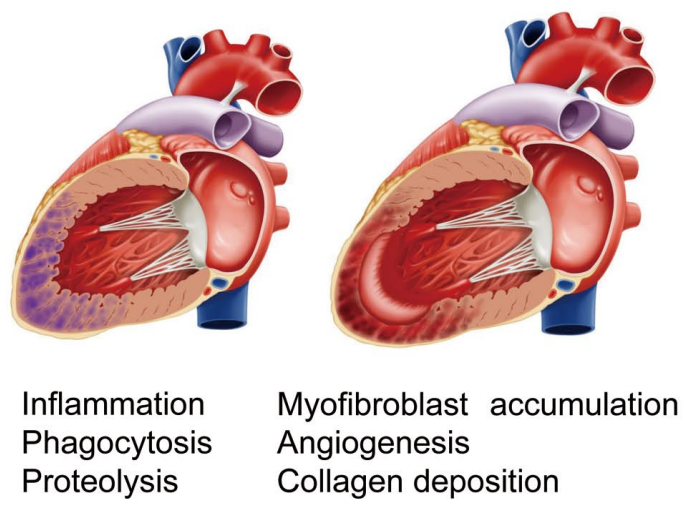

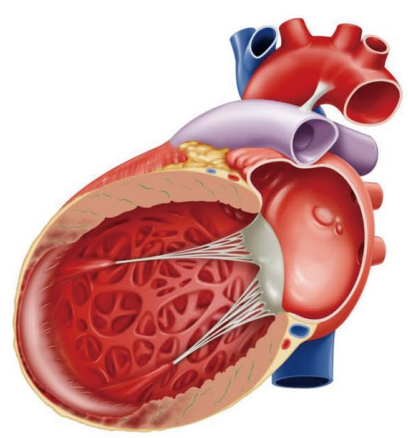

Significant changes in LV geometry and function
Figure 5. Adverse left ventricular (LV) remodeling after myocardial infarction. Aging and the presence of significant comorbidities, such as hypertension, dyslipidemia, diabetes, and chronic kidney disease, all impair the normal healing process after myocardial infarction and exacerbate remodeling. the main determinant of morbidity and mortality after MI. At the present time, therapeutic options to prevent LV remodeling are limited (Figure 5). Although the crucial roles of infiltrating immune cells and proinflammatory cytokines in the extent of myocardial damage and post-MI remodeling are well appreciated, the subsets of immune cells and types of inflammatory mediators that could modify the remodeling process remain undefined. Attempts at introducing nonselective antiinflammatory glucocorticoids for the treatment of acute MI have produced conflicting results. ${ }^{33,34}$ However, there is a concern that impairment of the acute wound healing process by glucocorticoids may facilitate wall thinning and ventricular rupture. To develop successful immunomodulatory therapies, we need to delineate the distinct subset of cells and cytokines that augment acute wound healing or are strongly involved in the persistent inflammation responsible for adverse LV remodeling.

To this end, my group created a large MI by permanent ligation of the coronary artery and identified a potential link between dendritic cells (DCs) and IL-17A-producing $\gamma \delta \mathrm{T}$ cells on late-stage $\mathrm{LV}$ remodeling after MI. The activated $\mathrm{CD} 11 \mathrm{c}^{+} \mathrm{MHC}-\mathrm{II}^{+} \mathrm{CD} 11 \mathrm{~b}^{+}$myeloid DCs originating from the bone marrow can infiltrate the post-MI heart and have potent immunoprotective properties during the post-MI healing process. ${ }^{35}$ IL-17A, which is almost exclusively produced by infiltrating $\gamma \delta T$ cells, was not involved in the acute inflammatory response, instead specifically functioning in late remodeling stages by promoting sustained infiltration of neutrophils and macrophages, stimulating macrophages to produce proinflammatory cytokines, aggravating cardiomyocyte death, enhancing fibroblast proliferation and pro-fibrotic gene expression. Thus, DC-mediated augmentation of healing or blockade of IL-17A-producing $\gamma \delta \mathrm{T}$ cells during hospitalization after $\mathrm{MI}$ is well worth planning for a clinical trial to evaluate whether these immunomodulatory therapies could prevent or ameliorate post-MI cardiac remodeling in humans.

\section{Acknowledgments}

It is my great honor and privilege to receive a SATO memorial award. I would like to express my enormous gratitude and appreciation to my mentors: Satoshi Ogawa (IUHW Mita Hospital), Keiichi Fukuda (Keio University School of Medicine), and Michael D. Schneider (Imperial College London) for their strong support and guidance. I would also like to acknowledge all my colleagues, friends, and collaborators all over the world. Having a good team is indispensable to accomplishing successful research.

\section{References}

1. The CONSENSUS Trial Study Group. Effects of enalapril on mortality in severe congestive heart failure: Results of the Cooperative North Scandinavian Enalapril Survival Study (CONSENSUS). N Engl J Med 1987; 316: 1429-1435.

2. Devereux RB, Wachtell K, Gerdts E, Boman K, Nieminen MS, Papademetriou V, et al. Prognostic significance of left ventricular mass change during treatment of hypertension. JAMA 2004; 292: $2350-2356$.

3. Pan J, Fukuda K, Kodama H, Makino S, Takahashi T, Sano M, et al. Role of angiotensin II in activation of the JAK/STAT pathway induced by acute pressure overload in the rat heart. Circ Res 1997; 81: $611-617$.

4. Kodama H, Fukuda K, Pan J, Makino S, Baba A, Hori S, et al. Leukemia inhibitory factor, a potent cardiac hypertrophic cytokine, activates the JAK/STAT pathway in rat cardiomyocytes. Circ Res 1997; 81: $656-663$.

5. Kodama H, Fukuda K, Pan J, Makino S, Sano M, Takahashi T, et al. Biphasic activation of the JAK/STAT pathway by angiotensin II in rat cardiomyocytes. Circ Res 1998; 82: 244-250.

6. Sano M, Fukuda K, Kodama H, Takahashi T, Kato T, Hakuno D, et al. Autocrine/paracrine secretion of IL-6 family cytokines causes angiotensin II-induced delayed STAT3 activation. Biochem Biophys Res Coтmun 2000; 269: 798-802.

7. Sano M, Fukuda K, Kodama H, Pan J, Saito M, Matsuzaki J, et al. Interleukin-6 family of cytokines mediate angiotensin II-induced cardiac hypertrophy in rodent cardiomyocytes. J Biol Chem 2000; 275: 29717-29723.

8. Harada M, Itoh H, Nakagawa O, Ogawa Y, Miyamoto Y, Kuwahara $\mathrm{K}$, et al. Significance of ventricular myocytes and nonmyocytes interaction during cardiocyte hypertrophy: Evidence for endothelin-1 as a paracrine hypertrophic factor from cardiac nonmyocytes. Circulation 1997; 96: 3737-3744.

9. Kuwahara K, Saito Y, Harada M, Ishikawa M, Ogawa E, Miyamoto $\mathrm{Y}$, et al. Involvement of cardiotrophin-1 in cardiac myocyte-nonmyocyte interactions during hypertrophy of rat cardiac myocytes in vitro. Circulation 1999; 100: 1116-1124.

10. Kanazawa H, Ieda M, Kimura K, Arai T, Kawaguchi-Manabe H, Matsuhashi T, et al. Heart failure causes cholinergic transdifferentiation of cardiac sympathetic nerves via gp130-signaling cytokines in rodents. J Clin Invest 2010; 120: 408-421.

11. Kimura K, Ieda M, Fukuda K. Development, maturation, and transdifferentiation of cardiac sympathetic nerves. Circ Res 2012; 110: $325-336$.

12. Olson EN, Schneider MD. Sizing up the heart: Development redux in disease. Genes Dev 2003; 17: 1937-1956.

13. Sano M, Abdellatif M, Oh H, Xie M, Bagella L, Giordano A, et al. Activation and function of cyclin t-Cdk9 (positive transcription elongation factor-b) in cardiac muscle-cell hypertrophy. Nat Med 2002; 8: $1310-1317$.

14. Yang Z, Zhu Q, Luo K, Zhou Q. The 7sk small nuclear RNA inhibits the Cdk9/cyclin t1 kinase to control transcription. Nature 2001; 414: $317-322$.

15. Nguyen VT, Kiss T, Michels AA, Bensaude O. 7SK small nuclear RNA binds to and inhibits the activity of $\mathrm{Cdk} 9 /$ cyclin $\mathrm{t}$ complexes. 
Nature 2001; 414: 322-325.

16. Sano M, Schneider MD. Cyclins that don't cycle: Cyclin T/cyclindependent kinase-9 determines cardiac muscle cell size. Cell Cycle 2003; 2: 99-104.

17. Sano M, Schneider MD. Cyclin-dependent kinase-9: An RNAPII kinase at the nexus of cardiac growth and death cascades. Circ Res 2004; 95: 867-876.

18. Sano M, Wang SC, Shirai M, Scaglia F, Xie M, Sakai S, et al. Activation of cardiac Cdk9 represses PGC-1 and confers a predisposition to heart failure. EMBO J 2004; 23: 3559-3569.

19. Sano M, Schneider MD. Energizer: PGC-1 alpha keeps the heart going. Cell Metab 2005; 1: 216-218.

20. Sano M, Izumi Y, Helenius K, Asakura M, Rossi DJ, Xie M, et al. Ménage-à-trois 1 is critical for the transcriptional function of PPARgamma coactivator 1. Cell Metab 2007; 5: 129-142.

21. Sano M, Tokudome S, Shimizu N, Yoshikawa N, Ogawa C, Shirakawa $\mathrm{K}$, et al. Intramolecular control of protein stability, subnuclear compartmentalization, and coactivator function of peroxisome proliferator-activated receptor gamma coactivator 1alpha. J Biol Chem 2007; 282: $25970-25980$.

22. Zhang Y, Sano M, Shinmura K, Tamaki K, Katsumata Y, Matsuhashi $\mathrm{T}$, et al. 4-hydroxy-2-nonenal protects against cardiac ischemia-reperfusion injury via the Nrf2-dependent pathway. J Mol Cell Cardiol 2010; 49: 576-586.

23. Takeuchi F, Isono M, Nabika T, Katsuya T, Sugiyama T, Yamaguchi $\mathrm{S}$, et al. Confirmation of ALDH2 as a major locus of drinking behavior and of its variants regulating multiple metabolic phenotypes in a Japanese population. Circ J 2011; 75: 911-918.

24. Endo J, Sano M, Katayama T, Hishiki T, Shinmura K, Morizane S, et al. Metabolic remodeling induced by mitochondrial aldehyde stress stimulates tolerance to oxidative stress in the heart. Circ Res 2009; 105: $1118-1127$

25. Sano M. Mitochondrial protection and the reversal of left ventricular remodeling. Circ J 2009; 73: 2017-2018.
26. Sano M. Cardioprotection by hormetic responses to aldehyde. Circ J 2010; 74: 1787-1793

27. Shiba N, Nochioka K, Miura M, Kohno H, Shimokawa H. Trend of westernization of etiology and clinical characteristics of heart failure patients in Japan: First report from the CHART-2 study. Circ J 2011; 75: $823-833$.

28. Velagaleti RS, Pencina MJ, Murabito JM, Wang TJ, Parikh NI, D'Agostino RB, et al. Long-term trends in the incidence of heart failure after myocardial infarction. Circulation 2008; 118: 2057-2062.

29. Yellon DM, Hausenloy DJ. Myocardial reperfusion injury. $N$ Engl $J$ Med 2007; 357: 1121-1135.

30. Ohsawa I, Ishikawa M, Takahashi K, Watanabe M, Nishimaki K, Yamagata $\mathrm{K}$, et al. Hydrogen acts as a therapeutic antioxidant by selectively reducing cytotoxic oxygen radicals. Nat Med 2007; 13: 688-694.

31. Hayashida K, Sano M, Ohsawa I, Shinmura K, Tamaki K, Kimura K, et al. Inhalation of hydrogen gas reduces infarct size in the rat model of myocardial ischemia-reperfusion injury. Biochem Biophys Res Commun 2008; 373: 30-35.

32. Yoshida A, Asanuma H, Sasaki H, Sanada S, Yamazaki S, Asano Y, et al. $\mathrm{H}_{2}$ mediates cardioprotection via involvements of KATP channels and permeability transition pores of mitochondria in dogs. Cardiovasc Drugs Ther 2012; 26: 217-226.

33. Giugliano GR, Giugliano RP, Gibson CM, Kuntz RE. Meta-analysis of corticosteroid treatment in acute myocardial infarction. Am J Cardiol 2003; 91: 1055-1059.

34. Tokudome S, Sano M, Shinmura K, Matsuhashi T, Morizane S, Moriyama H, et al. Glucocorticoid protects rodent hearts from ischemia/reperfusion injury by activating lipocalin-type prostaglandin D synthase-derived PGD2 biosynthesis. J Clin Invest 2009; 119: 1477 1488.

35. Anzai A, Anzai T, Nagai S, Maekawa Y, Naito K, Kaneko H, et al. Regulatory role of dendritic cells in postinfarction healing and left ventricular remodeling. Circulation 2012; 125: 1234-1245. 